\title{
Archives
}

\section{Les archives définitives à la Division de la gestion de documents et des archives de l'Université de Montréal : accroissement, traitement, diffusion}

\section{MICHEL CHAMPAGNE}

Volume 46, numéro 2, 2017

$50^{\mathrm{e}}$ anniversaire de la DGDA

URI : https://id.erudit.org/iderudit/1040380ar

DOI : https://doi.org/10.7202/1040380ar

Aller au sommaire du numéro

Éditeur(s)

Association des archivistes du Québec (AAQ)

ISSN

0044-9423 (imprimé)

2369-9256 (numérique)

Découvrir la revue

Citer cet article

Champagne, M. (2017). Les archives définitives à la Division de la gestion de documents et des archives de l'Université de Montréal : accroissement, traitement, diffusion. Archives, 46(2), 61-80. https://doi.org/10.7202/1040380ar
Résumé de l'article

Le texte présente la gestion des archives définitives à la Division de la gestion de document et des archives de l'Université de Montréal, l'un des éléments essentiels du mandat de cette unité. Après avoir situé les interventions de la Division de la gestion de documents et des archives dans le cadre de la Politique sur la gestion de documents et des archives de l'Université de Montréal, le texte aborde la question de l'accroissement des archives. Sont présentés tour à tour, l'impact du calendrier de conservation sur la conservation des archives institutionnelles et le processus d'acquisition des archives de sources privées. Par la suite, le texte décrit le traitement des archives définitives, tant le traitement préliminaire que le traitement définitif. Enfin, l'article brosse un tableau des différentes actions de la Division en ce qui concerne la diffusion des archives soit la référence, les expositions, les réseaux sociaux et d'autres projets de diffusion. 


\section{ANNIVERSAIRE DE LA DGDA}

\section{Les archives définitives à la Division de la gestion de documents et des archives de l'Université de Montréal: accroissement, traitement, diffusion}

\section{MICHEL CHAMPAGNE}

Archiviste, Division de la gestion de documents et des archives, Université de Montréal

\section{INTRODUCTION}

Sur la page d'accueil du site Web de la Division de la gestion de documents et des archives (DGDA) de l'Université de Montréal, on retrouve cette phrase: «Pour une mémoire institutionnelle d'utilité courante et historique ". Cette vision est présente chez mes collègues Taïk Bourhis et Nathalie Denis qui ont traité de la gestion des archives courantes et intermédiaires. Pour ma part, je vais la poursuivre en abordant la question des archives définitives au sein de la DGDA. Pour paraphraser le titre d'un film connu, j'aimerais présenter « tout ce que vous avez toujours voulu savoir sur les archives définitives à la DGDA (sans jamais oser le demander)». Pour 
ce faire, je vais vous présenter les actions de la DGDA liées à l'acquisition, au traitement et à la diffusion des archives définitives. Je tiens à remercier ma collègue Monique Voyer, responsable de la référence, qui m'a indiqué les éléments à aborder en ce qui concerne la diffusion des archives définitives.

\section{LE MANDAT DE LA DGDA ET LES ARCHIVES DÉFINITIVES}

Les archives définitives, qu'elles soient institutionnelles ou de sources privées, font partie intégrante de la mission de la Division de la gestion de documents et des archives de I'Université de Montréal parce que la DGDA, c'est aussi :

- 9761 contenants d'archives définitives;

- 11760 dossiers d'archives photographiques traités de façon définitive;

- plus de 11200 documents audiovisuels traités de façon préliminaire ou définitive;

- plus de 26300 documents d'archives imprimées.

La DGDA est chargée de la conservation, de l'accessibilité et de la mise en valeur des archives historiques produites par les unités de I'Université de Montréal. De plus, «La Division acquiert aussi des archives de source privée qui sont jugées nécessaires à l'enseignement, à la recherche, et à l'histoire de l'institution. »' Ce mandat repose sur la Politique sur la gestion de documents et des archives de l'Université de Montréal qui indique entre autres que I'Université a comme objectif: de conserver et faire connaître les documents qu'elle possède qui ont une valeur patrimoniale et de faire connaître l'histoire de l'Université de Montréal.

Pour atteindre ces objectifs, la Division a un certain nombre de responsabilités définies dans la politique sur la gestion de documents et des archives. Par exemple, on y précise que la DGDA:

- Élabore et tient à jour les règles de conservation relatives à tout document produit ou reçu par une unité de I'Université ou par une personne à son emploi, dans le cadre de ses fonctions, quel que soit le support sur lequel il se trouve ou a été créé ou le format dans lequel il se trouve ou a été créé.

- Traite de manière archivistique tout document que I'Université possède et qui a une valeur patrimoniale. 
- Procède à l'acquisition et au traitement archivistique de tout document de tiers que l'Université souhaite acquérir et qui a une valeur patrimoniale, dont les fonds d'archives privées.

- Communique, diffuse et met en valeur les documents de l'Université qui ont une valeur patrimoniale afin de faire connaître l'Université et de contribuer à l'acquisition de connaissances par le plus grand nombre de personnes possible 2 .

C'est sur la base de ces énoncés que s'articulent les pratiques de la Division en ce qui concerne la gestion des archives définitives.

\section{ACQUISITION, ACCROISSEMENT ET ÉVALUATION DES ARCHIVES DÉFINITIVES}

Les archives définitives conservées par la Division de la gestion de documents et des archives se composent à la fois d'archives institutionnelles ayant fait l'objet de versements et d'archives privées qui ont fait l'objet d'une acquisition. D'ailleurs, il serait sans doute plus précis de parler $d^{\prime}$ 'accroissement quand il est question des processus et procédures par lesquels s'enrichissent les fonds et collections au sein de la DGDA. L'accroissement des archives est intimement lié à leur évaluation puisque les archives qui seront conservées de façon définitive sont celles qui, en principe, ont fait l'objet d'une évaluation permettant de déterminer les documents qui doivent être sauvegardés de façon permanente. Voyons concrètement comment cela s'articule.

\subsection{Archives institutionnelles}

À la DGDA, c'est le calendrier de conservation qui détermine quels documents feront l'objet d'un versement. L'évaluation des archives institutionnelles est donc réalisée dans le cadre de l'application des règles de conservation des documents. Ce sont les règles de conservation qui déterminent, entre autres, quels documents seront éliminés et quels documents seront conservés de façon permanente. D'ailleurs, lors de I'élaboration de nouvelles règles ou lors de la modification de règles existantes, la valeur d'information et de témoignage des documents est toujours prise en compte pour déterminer le statut final des documents.

Mais quels sont les documents institutionnels conservés de façon définitive? 


\subsubsection{La mission}

II s'agit tout d'abord de documents liés aux missions centrales de I'Université, soit l'enseignement et la recherche. En ce qui concerne I'enseignement, on vise à documenter tant ses aspects administratifs que méthodologiques ou pédagogiques. C'est ainsi que, parmi les documents conservés de façon permanente, on retrouve, entre autres, des archives associées à l'organisation de l'enseignement et à sa gestion courante. II y a aussi des archives liées aux programmes d'études, aux cours et à l'évaluation des études. II s'agit en partie de documents associés à des processus de gestion; sont donc conservés les politiques et procédures, les règlements, les documents qui définissent les principes et les règles encadrant l'enseignement. Sont aussi conservés les documents concernant les structures organisationnelles de l'enseignement (création et organisation des facultés, écoles, départements et autres). II s'agit donc de documents permettant de dégager une vue d'ensemble de la gestion de l'enseignement. Les documents associés aux programmes et aux cours donnent quant à eux une vision plus précise et détaillée de ce qui est enseigné. Par exemple, les dossiers de création et de modification de programmes témoignent d'un consensus pour une période donnée sur le contenu de la formation associé à un champ de connaissance. Qu'est-ce qui sera enseigné, selon quelle séquence et avec quels objectifs? Pour leur part, les dossiers de cours qui incluent, entre autres, les plans de cours constituent un élément central pour documenter l'enseignement. Ils permettent d'avoir une vision encore plus précise et détaillée de la réalité de l'enseignement puisque le cours est le lieu où l'activité d'enseignement se réalise concrètement. De plus, pris dans leur ensemble, les documents liés aux dossiers de cours témoignent de l'évolution du contenu des cours et donc, de l'évolution de l'enseignement.

En ce qui concerne la recherche et la création, les documents conservés documentent tant leurs aspects administratifs que méthodologiques. Ils permettent de comprendre les processus associés à l'organisation et à la gestion de la recherche en dégageant des vues d'ensemble et des orientations. Comme pour l'enseignement, les politiques et procédures liées à la recherche sont conservées ainsi que les documents relatifs à la mise sur pied et à la réalisation des activités des unités de recherche de I'UdeM (centres, laboratoires, groupes, instituts, chaires). Rappelons que l'intervention de I'Université en matière de recherche se fait en grande partie par ses unités. Parmi les autres documents associés à la 
recherche qui sont conservés, on retrouve des documents associés à l'éthique et aux normes déontologiques à respecter lors de la réalisation de projets de recherche, ainsi qu'au financement de la recherche par I'Université. Certains documents associés aux projets réalisés par une unité de recherche sont aussi conservés de façon permanente. Toutefois, une partie importante des archives produites dans le cadre de la recherche échappent au calendrier de conservation puisqu'elles sont sous la responsabilité personnelle de professeurs/chercheurs. En effet, ce sont eux et non l'Université qui dans un grand nombre de cas gèrent leurs projets de recherche et reçoivent des subventions d'organismes externes. Ce sont donc des archives privées.

\subsubsection{Les personnes}

Les activités d'enseignement et de recherche de même que les autres activités en lien avec le fonctionnement de l'Université sont réalisées pour et par des personnes: étudiants, personnel enseignant et non enseignant. II n'est donc pas étonnant que parmi les archives définitives conservées par la Division de la gestion de documents et des archives on retrouve des documents associés à ces individus. II s'agit moins des différents types de dossiers individuels qui, en raison de leur contenu ont des périodes semi-actives très longues et dont l'accès est encadré de façon très stricte, mais plutôt des documents associés aux personnes en tant que groupe ou touchant un ensemble de personnes. Par exemple, dans le cas des documents relatifs à la clientèle étudiante et à son cheminement scolaire sont, entre autres, conservés les documents relatifs aux activités de promotion organisées dans le but de recruter de nouveaux étudiants (rapports décrivant les activités organisées et les résultats obtenus). Sont aussi versés les statistiques, études et rapports officiels relatifs au suivi de l'évolution de la clientèle étudiante de I'UdeM. Certains documents associés aux relations entre l'Université de Montréal et les regroupements associatifs étudiants sont conservés, entre autres, les dossiers de reconnaissance d'associations et les dossiers d'activités organisées conjointement par les associations étudiantes et les unités de l'Université.

Les documents relatifs à la gestion du personnel administratif et du personnel enseignant (incluant le personnel de recherche) de l'UdeM font légalement l'objet de règles de conservation qui permettent d'en conserver les plus significatifs. C'est ainsi que sont conservées des archives associées 
à la planification des effectifs et des postes de I'UdeM, de même que celles reliées au recrutement et à l'embauche du personnel. Les interactions entre I'Université et son personnel sont aussi documentées par la conservation de documents créés pour la formation des différentes catégories de personnel et par celle des documents reliés aux relations de travail (entres autres, les dossiers d'accréditation de travail et de convention collective).

\subsubsection{Gestion des ressources, des actions de communication et des documents stratégiques}

Une université, c'est aussi des ressources financières, matérielles et informationnelles. C'est pourquoi des documents significatifs associés à la gestion de ces ressources sont conservés. L'Université de Montréal communique aussi avec les membres de la communauté tout comme avec la société dans laquelle elle évolue. Elle offre également des services à la communauté universitaire et à la société. Il est, par conséquent, important de conserver des traces de ces exercices de communication et de ces services offerts. C'est ce que la DGDA fait. Le calendrier de conservation identifie donc des documents à conservation permanente associés à ces activités. De plus, il détermine des documents stratégiques qui doivent être conservés quelles que soient les activités ou personnes auxquelles ils sont associés. Je n'en ferai pas une liste exhaustive, toutefois signalons-en quelquesuns. Font naturellement partie des archives définitives, les procès-verbaux des différentes instances universitaires (autant celles touchant l'ensemble de l'institution qu'une de ses unités), les contrats qui lient l'Université et ses composantes à d'autres parties, les rapports qui souvent concentrent et synthétisent l'information sur une question (par exemple les rapports annuels ou les rapports réalisés à la suite d'enquêtes ou de débats), les statistiques qui permettent d'illustrer en chiffres la vie de l'Université.

Les archives institutionnelles sont diverses et elles font l'objet d'une évaluation constante pour déterminer, entre autres, quels sont les documents qui permettront de mieux illustrer et comprendre l'Université de Montréal, son histoire, son fonctionnement, son évolution et ses orientations.

\subsection{Les archives privées}

Comme je l'ai mentionné, les archives définitives conservées par la Division de la gestion de documents et des archives comprennent 
également des archives privées qui ont fait l'objet d'une acquisition. Comme son mandat le permet, la DGDA acquiert des archives privées de diverses natures et sur différents supports. Les archives privées contiennent des documents textuels tels que de la correspondance, des rapports, des manuscrits, des imprimés. Dans ce dernier cas, il s'agit de publications (monographies et articles ou autres) produites par les créateurs des fonds dont on fait l'acquisition. La DGDA acquiert aussi des documents architecturaux, des documents cartographiques, des documents d'images en mouvement (films et vidéos), des documents sonores, des documents iconographiques tels que des dessins, des photographies, des ensembles multi-supports, des microformes. La DGDA acquiert également depuis quelques années des documents numériques de toute nature. À quelques exceptions près, la Division n'acquiert pas d'objet.

Les activités d'acquisition d'archives privées sont réalisées dans un cadre législatif et réglementaire bien défini. Les lois qui encadrent le processus d'acquisition sont bien connues des archivistes. II s'agit, entre autres, de la Loi québécoise sur les archives, de la Loi sur le patrimoine culturel, de la Loi sur l'accès aux documents des organismes publics et sur la protection des renseignements personnels et de la Loi sur les impôts. Certaines lois fédérales ont également un impact sur nos acquisitions: la Loi sur le droit d'auteur, celle sur l'exportation et l'importation des biens culturels de même que la Loi de l'impôt sur le revenu.

Certains principes encadrent notre processus d'acquisition:

- L'acquisition se justifie d'abord et avant tout par la signification et la valeur des documents offerts dans l'atteinte de l'un ou l'autre des objectifs visés par le processus d'acquisition retenu.

- Toute acquisition peut être sujette à une réévaluation.

- L'acquisition se fait dans le respect de toute législation touchant la pratique archivistique québécoise et canadienne.

- L'acquisition se fait dans le respect des principes archivistiques généralement reconnus, tels le principe de respect des fonds et le principe de provenance territoriale.

- La Division de la gestion de documents et des archives évite, dans la mesure du possible, le fractionnement des fonds. 
- L'acquisition tient compte des politiques d'acquisition des autres services d'archives, notamment ceux des autres universités et ceux des institutions de la région de Montréal.

- L'acquisition se fait en tenant compte de la capacité de I'Université à les rendre accessibles; cette capacité dépend, entre autres, de l'état de conservation et du volume des documents concernés, des conditions d'acquisition et des ressources financières et humaines nécessaires pour traiter et conservés les documents à acquérir.

La Division de la gestion de documents et des archives vise des objectifs précis lorsqu'elle acquiert des fonds d'archives privées. Le premier objectif est de compléter la mémoire institutionnelle nécessaire à la compréhension de la mission, des valeurs et des principes fondateurs de l'Université, à l'interprétation de ses traditions, de ses orientations, de ses politiques et de ses activités tant sur le plan de son administration que de celui de l'enseignement et de la recherche. II s'agit donc ici de participer à la documentation de I'histoire de l'Université de Montréal. Rappelons que I'Université a été créée en 1878 et la Division de la gestion de documents et des archives en 1966. L'UdeM a connu de multiples déménagements au cours de son histoire, ce qui n'a pas nécessairement eu un effet positif sur la conservation des archives. Les documents produits par les unités n'ont pas toujours été gérés avant la création de la DGDA à partir de principes professionnels reconnus ce qui a pu également entraîner des pertes de documents. Les archives privées peuvent, dans certains cas, combler ces lacunes. Elles peuvent aussi donner un éclairage différent sur certains événements de I'histoire de I'Université. Le second objectif est de répondre aux besoins, en matière d'enseignement et de recherche, dans les domaines qu'entend privilégier l'Université et identifiés par elle.

En matière d'acquisition d'archives privées, I'Université accorde la priorité aux fonds et aux collections d'archives associées ou quasi institutionnelles, c'est-à-dire essentiellement d'archives provenant de la communauté universitaire. II s'agit de fonds ou collections de membres du personnel enseignant et non enseignant ou de chercheurs de l'Université. La DGDA acquiert aussi des documents de diplômés et d'étudiants, de regroupements tels que les associations étudiantes et les syndicats représentant les employés de l'Université. Nous acceptons également des documents d'organismes dont les activités sont étroitement liées à celles de l'institution (dont le lieu principal d'activité se trouve sur le campus 
ou dont les membres sont liés à la communauté universitaire). Parmi les archives associées ou quasi institutionnelles, nous retrouvons aussi des documents sur la vie de l'Université, de ses unités et des personnes qui y ont œuvré, mais qui proviennent de personnes non associées à I'UdeM ou d'organismes externes.

La DGDA acquiert également, en tenant compte des ressources humaines et matérielles requises pour la conservation, le traitement et la mise en valeur de ces documents, des fonds et des collections d'archives privées de personnes ou d'organismes lorsqu'ils constituent une source d'information importante pour l'enseignement, la recherche liée à certains domaines et, dans certains cas, pour compléter des fonds et des collections déjà acquis.

Les fonds d'archives privées susceptibles d'être acquis dans ce contexte sont:

- des fonds d'archives dans le domaine de l'éducation;

- des fonds d'archives en musique;

- des fonds d'archives touchant le domaine des sciences, y compris des sciences de la santé, dont la médecine;

- des fonds d'archives littéraires.

Les modes d'acquisition privilégiés sont le don et le legs. En théorie, la DGDA peut accepter des archives définitives en dépôt, en prêt ou encore avoir recours à l'achat. Toutefois, en pratique, le dépôt, le prêt et l'achat n'ont pas été utilisés depuis de très nombreuses années.

Les acquisitions d'archives privées font en général l'objet d'une entente contractuelle. Celle-ci prend habituellement la forme d'une convention d'acquisition précisant les droits et les obligations des deux parties, les conditions particulières concernant I'utilisation du fonds, notamment les restrictions de consultation et de reproduction de documents. La convention doit contenir une déclaration relative à la propriété des droits d'auteur. Une description sommaire des documents faisant l'objet de l'acquisition est annexée à la convention.

Les archives définitives conservées par la DGDA, tant les archives institutionnelles que privées, constituent un patrimoine culturel important. Le processus de leur entrée à la DGDA, on vient de le voir, est régi par 
un ensemble de politiques et d'outils qui respectent les règles de l'art. Mais une fois qu'ils sont à la Division, que fait-on avec ces documents?

\section{LE TRAITEMENT DES ARCHIVES DÉFINITIVES À LA DGDA}

Les archives définitives sélectionnées par la DGDA dans le cadre de versement $d$ 'archives institutionnelles ou d'acquisition d'archives privées sont conservées en vue d'une éventuelle utilisation. Pour être utilisées, elles doivent être connues et aisément repérables. Différentes opérations de description sont réalisées par la DGDA pour produire des outils de repérage permettant d'atteindre ces buts. La création d'outils de repérage est au cœur du traitement des archives définitives. Le traitement des archives définitives peut aussi, selon les cas, entraîner des opérations de tri, de classification, de classement et d'indexation. De plus, des besoins particuliers peuvent nécessiter certaines actions pour assurer la préservation des documents à conservation permanente. À la DGDA, comme dans d'autres services d'archives, deux types de traitement peuvent être effectués: le traitement préliminaire ou le traitement définitif. La politique de la Division est de réaliser le traitement préliminaire de toutes les archives définitives dont elle a la garde. En ce qui concerne le traitement définitif, il est réservé à un nombre plus restreint de documents. Mais voyons de plus près en quoi consistent ces traitements.

Pour présenter rapidement le traitement préliminaire à la DGDA, et éviter une présentation fastidieuse des processus et procédures de traitement, j'ai décidé d'insister sur les unités de traitement et les outils qui y sont associés.

\subsection{Le traitement préliminaire}

Comme je le mentionnais, toutes les archives définitives connaissent un traitement préliminaire, tant les archives institutionnelles que les archives privées. Pour les archives institutionnelles, le traitement préliminaire encadre le changement d'état d'archives courantes à archives définitives et d'archives intermédiaires à archives définitives. Pour les archives privées, le traitement préliminaire est associé à la prise de possession de ces documents par la Division de la gestion de documents et des archives. Certaines opérations sont identiques dans les deux cas. Par contre, des particularités peuvent entraîner des actions spécifiques. Dans le cadre du 
traitement préliminaire, la DGDA utilise différentes unités de traitement. Ces unités sont le contenant, le versement ou l'acquisition et le fonds.

Constituant la première unité de traitement, chaque contenant d'un versement ou d'une acquisition fait l'objet d'une description réalisée sur un bordereau. Ce bordereau est un document Word dont les versions numériques et imprimées seront utilisées. Le bordereau employé pour les archives institutionnelles est le même que celui utilisé pour le transfert des archives intermédiaires. Un bordereau similaire, mais non identique, est employé pour les archives privées. Chaque bordereau de description contient des informations sur la provenance des documents, soit le nom et la cote du fonds de l'unité administrative, de l'organisme ou de la personne qui a produit ou reçu les documents décrits. II comprend aussi des informations sur les supports et les types de documents présents dans la boîte de même qu'un numéro unique attribué au contenant, une adresse de conservation correspondant à la localisation de la boîte dans les magasins d'archives de la DGDA et le numéro du versement ou de l'acquisition auquel il est associé. Une description des dossiers que I'on retrouve dans le contenant complète le bordereau, tant celui des archives institutionnelles que celui des archives privées. II s'agit d'une liste souvent détaillée des dossiers. Selon la nature homogène ou hétérogène des dossiers, ils sont identifiés par leurs titres spécifiques (par exemple DIN 4010 - Atelier de design 6 - Tryptique - Exploration ou par un identifiant général comme Procès-verbaux et documents afférents du comité spécial sur l'évaluation de l'enseignement). En plus de ces informations, chaque bordereau inclut également le nombre de dossiers associés à chaque titre ou à chaque identifiant de même que les années inclusives des documents contenus dans les dossiers. Les bordereaux décrivant les archives institutionnelles contiennent en général les règles conservation qui s'appliquent à chaque dossier.

Les bordereaux pour les archives institutionnelles sont réalisés par le personnel de la DGDA lorsque les documents proviennent des dépôts de la DGDA, à la suite de l'application du calendrier de conservation sur des séries de contenants. Quand il s'agit de versements provenant directement des unités, c'est le personnel de l'unité qui prépare les bordereaux. Dans ce dernier cas, une vérification est réalisée, par la DGDA, pour s'assurer que le bordereau corresponde bien au contenu de la boîte qu'il décrit et que les bonnes règles de conservation ont été appliquées. Les bordereaux 
des contenants d'archives privées sont quant à eux complétés par les membres du personnel de la DGDA ou par des stagiaires. Selon la situation qui se présente, ils peuvent être rédigés chez le donateur des documents ou dans les bureaux de la DGDA.

Lors du traitement préliminaire, chaque bordereau est imprimé et son fichier est importé dans le logiciel de gestion intégrée des documents SyGED. Le bordereau imprimé avec les autres bordereaux du même versement ou de la même acquisition est ajouté aux bordereaux du fonds à l'origine des documents. Les bordereaux résultant du traitement préliminaire forment le premier instrument de recherche et parfois le seul, remis aux chercheurs, si aucun traitement définitif n'est réalisé. II s'agit d'outils de repérage essentiels pour les chercheurs puisqu'ils n'ont pas accès à SyGED.

Un versement ou une acquisition (seconde unité de traitement) se compose d'un ensemble de documents regroupés dans un ou plusieurs contenants. Chaque versement ou acquisition reçoit un numéro d'identification spécifique formé de l'année universitaire suivi d'un numéro séquentiel; par exemple le numéro séquentiel 2015-2016-26 correspond au $26^{\mathrm{e}}$ versement ou à la $26^{\mathrm{e}}$ acquisition traitée durant l'année universitaire 2015-2016. II n'y a pas de distinction ici entre les archives institutionnelles et les archives privées.

Chaque versement ou acquisition est décrit à l'intérieur du Registre des acquisitions et versements qui est l'une des composantes du logiciel SyGED. II s'agit d'une description sommaire de l'ensemble des contenants et documents qui forment un versement ou une acquisition. Comme éléments de description, on retrouve principalement la nature de l'accroissement (versement ou acquisition), un titre correspondant au numéro d'acquisition, une description prenant la forme de la portée/contenu du versement ou de l'acquisition, les dates extrêmes de même qu'une collation des documents. La description comprend également différentes notes.

Les informations que l'on retrouve dans une notice descriptive d'un versement ou d'une acquisition constituent une synthèse utile pour la préparation de statistiques de traitement pour la production de l'état général. La création ou la modification d'une notice de l'état général des fonds, identifié sous l'appellation Catalogue des archives, est la dernière étape du traitement préliminaire. II présente une description de chaque fonds d'archives contenant des archives définitives. II s'agit d'une 
description normalisée basée sur les Règles de description des documents $d$ 'archives (RDDA). C'est souvent après avoir consulté ces descriptions que les chercheurs nous contactent, puisqu'elles sont accessibles à partir de notre site Web et qu'elles constituent la première description accessible.

\subsection{Traitement définitif}

Dans le cadre de ses activités, la DGDA effectue le traitement définitif de certains fonds. Comme les archivistes le savent, le traitement définitif vise à compléter l'organisation, la classification, le tri et la description de certains fonds. II s'agit de fournir au chercheur l'instrument de recherche le plus détaillé possible. À la DGDA, le traitement définitif a aussi comme objectif de décrire et d'indexer certains types de documents, plus spécifiquement les archives photographiques et les archives imprimées.

La réalisation du traitement définitif demande en général plus de temps et des ressources importantes. La Division de la gestion de documents et des archives, à l'instar de la grande majorité des services $d^{\prime}$ archives, ne peut réaliser ce type de traitement pour tous les fonds d'archives dont elle a la responsabilité. Des choix et des priorités de traitement doivent être établis. Ces choix sont basés d'abord sur les besoins de la recherche et sont faits, entre autres, en identifiant les fonds consultés par les chercheurs, mais qui n'ont pas fait l'objet d'un traitement définitif. Les ressources nécessaires et disponibles pour réaliser le traitement forment l'autre élément clé dans la détermination des fonds qui feront l'objet d'un traitement définitif. Avant d'entreprendre ce type de traitement, nous nous assurons que nous avons le personnel et le matériel nécessaires et que nous disposons du temps requis pour réaliser un traitement de qualité.

Le traitement d'un fonds dans son ensemble est réalisé par le personnel de la DGDA, des contractuels ou des stagiaires. II implique différentes étapes identiques en grande partie à celles réalisées par d'autres services d'archives. Il y a tout d'abord l'établissement d'une classification, si une classification préalable n'est pas présente. On passe par la suite au classement physique des unités de rangement (les dossiers) à moins que l'ordre existant corresponde à l'ordre original des documents et aux besoins de la recherche. Après le classement, quelques opérations sont réalisées concurremment. II s'agit du tri final des documents, de leur description et de leur indexation. Chaque unité de description, en général le dossier, 
fera l'objet d'une seule analyse permettant de déterminer si l'unité doit être conservée, d'identifier les éléments qui devront se retrouver dans sa description et d'identifier les concepts et les termes d'indexation à retenir. La description est établie en fonction des RDDA. Quant à l'indexation, elle fait l'objet d'un contrôle du vocabulaire. Les descriptions réalisées de même que les termes d'indexation sont saisis dans le logiciel SyGED, soit au moment de l'analyse des dossiers ou ultérieurement. Le traitement définitif d'un fonds à la DGDA inclut certaines opérations liées à la préservation; par exemple les trombones sont enlevés, les documents papier sont mis dans des chemises sans acide et tous les documents sont mis dans des contenants sans acide.

Puisque le traitement définitif vise à fournir un instrument de recherche aux chercheurs, on génère à partir du logiciel SyGED un rapport contenant les notices descriptives d'un fonds traité. Ce rapport est accessible sur le site Web de la DGDA à partir de la notice du fonds dans le Catalogue des archives. Finalement, chaque fonds ayant fait l'objet d'un traitement définitif sera par la suite numérisé ${ }^{3}$.

Certains types de documents peuvent aussi faire l'objet d'un traitement définitif, même si ce n'est pas le cas pour les autres documents du fonds auquel ils appartiennent. II s'agit principalement des archives photographiques et des archives imprimées. Les archives photographiques, c'est bien connu, suscitent un fort intérêt chez plusieurs catégories d'utilisateurs d'archives. Cet intérêt est à l'origine de nombreuses demandes reçues par les services d'archives et la Division de la gestion de documents et des archives n'est pas étrangère au phénomène. C'est ainsi que depuis de nombreuses années, la DGDA réalise le traitement définitif de photographies incluses ou non dans le traitement définitif d'un fonds. Le traitement définitif des archives photographiques comprend le tri des documents, leur description basée sur les RDDA, leur indexation ${ }^{4}$ de même que le rangement des documents dans des contenants protecteurs. Les archives imprimées, quant à elles, sont décrites et indexées à la pièce. Les informations ainsi générées sont saisies dans I'application SyGED.

\subsection{Le traitement des archives définitives avec SyGED}

SyGED, I'application de gestion intégrée des archives utilisée par la Division de la gestion de documents et des archives, est au cœur de nos activités. Comme il a déjà été évoqué, elle joue un rôle central dans le 
traitement des archives à conservation permanente. En ce qui concerne le traitement préliminaire, les bordereaux réalisés à cette étape sont importés dans SyGED, ce qui permet la recherche en texte intégral. Quant au Registre des acquisitions et versements, il fait partie intégrante de SyGED et permet de contrôler les accroissements de documents. Lors du traitement définitif, toutes les notices descriptives sont saisies dans SyGED. Il est ensuite possible de générer des instruments de recherche accessibles par l'intermédiaire du site Web de la DGDA. Seule ombre au tableau, mais elle est importante pour nous, les chercheurs externes ne peuvent pas interroger SyGED et les chercheurs internes ne peuvent faire de recherches que sur les documents des unités auxquels ils sont associés.

\section{LA DIFFUSION DES ARCHIVES DÉFINITIVES}

Si des documents sont versés, acquis et traités par la Division de la gestion de documents et des archives, c'est bien sûr pour travailler à la constitution et à la conservation de la mémoire de l'Université de Montréal et de la société dans laquelle elle évolue. Mais cette mémoire n'est pas une mémoire morte. C'est une mémoire vive et les documents qui la composent sont diffusés de plusieurs manières. La diffusion des archives définitives à la DGDA prend plusieurs formes: référence, expositions, présence sur les réseaux sociaux et autres projets, tout est fait pour que la mémoire conservée soit vraiment d'utilité courante.

\subsection{La référence}

La référence à la DGDA est assurée par une archiviste professionnelle. Son rôle est de répondre aux demandes internes d'information et de recherche, d'assister les chercheurs à l'aide des instruments de recherche, de base de données et des réseaux de diffusion. Elle doit également répondre aux demandes externes d'information, de recherche et de reproduction de documents. Les clientèles qui ont recours aux services de la référence sont variées. II y a d'abord la clientèle interne formée par les directions de l'Université, des facultés et des autres unités de I'Université. Ce type de clientèle comprend également les étudiants, les professeurs, et les autres employés de I'Université. Quant à la clientèle externe, elle regroupe des chercheurs provenant de milieux variés (professeurs, étudiants, journalistes, citoyens, etc.) et des organismes divers (maisons d'édition, maison de production, musées, etc.). 
Ces clientèles sont à l'origine d'une intense activité ; en voici quelques exemples chiffrés. Durant I'année universitaire 2014-2015, I'archiviste responsable de la référence a répondu à 470 demandes de recherche ou de renseignement. Durant la même période, 329 personnes ont été accueillies à notre salle de consultation, 430 fonds ont été consultés et 15332 documents ont été reproduits.

En 2014-2015 la référence a permis à ses clientèles:

- de répondre à des questions liées au fonctionnement de l'Université. Par exemple, I'historique de la création ou de la modification d'un programme;

- d'écrire des articles, des livres, des mémoires de maîtrise et des thèses de doctorat. Par exemple, un livre sur la visite du général Charles de Gaulle en 1967 à Montréal ;

- de réaliser des films, des vidéos et des émissions de télévision. Par exemple, une vidéo sur Hans Selye;

- de présenter des conférences. Par exemple, une conférence présentée dans le cadre $50^{\mathrm{e}}$ anniversaire de l'ouverture de la salle Claude-Champagne;

- d'organiser des expositions. Par exemple, une exposition sur le $50^{\mathrm{e}}$ anniversaire de la Faculté des sciences de l'éducation de I'Université de Montréal ;

- de produire des affiches et des sites Web. Par exemple, une affiche concernant l'histoire du féminisme à l'UdeM.

\subsection{Expositions}

Depuis de nombreuses années, la DGDA participe à des expositions à titre de contributeur ou de maître d'œuvre. Avec l'arrivée d'Internet, la réalisation d'exposition a pris une nouvelle forme. Depuis 1999, la Division de la gestion de documents et des archives rend disponible à partir de son site Web des expositions virtuelles abordant différents sujets. Au total, quinze expositions ont été présentées. Quatorze de ces expositions sont toujours accessibles à partir du site Web de la DGDA. Une seule a été retirée parce qu'elle était redondante avec une exposition plus récente. 
Les expositions abordent des sujets diversifiés ce qui nous permet d'illustrer la richesse de nos fonds et collections. C'est ainsi que I'on retrouve des expositions sur:

- des personnes: Louis Hémon, Édouard Montpetit, le frère MarieVictorin, Émile Olivier, Hans Selye;

- I'histoire de l'Université de Montréal: la Faculté des sciences, les chartes qui ont encadré le fonctionnement de l'Université de Montréal;

- I'histoire du Canada et du Québec: la Collection Baby;

- les sciences biologiques: récolter pour la science (biologie végétale), la poule Chanteclerc;

- le territoire québécois: Marie-Victorin au Québec;

- Noël tel qu'illustré dans le journal étudiant Le Quartier Latin.

Les expositions présentées par la DGDA sont de taille et de complexité variables. Certaines ne sont composées que de quelques pages Web alors que d'autres comprennent des centaines de fichiers. Cette variété est liée à la fois à l'origine des projets d'expositions et aux ressources déployées pour les réaliser. Certaines expositions sont le fruit de l'obtention de subventions d'organismes québécois ou canadiens (Bibliothèque et Archives nationales du Québec, Patrimoine canadien). D'autres ont été réalisées dans le cadre d'un cours de l'École de bibliothéconomie et des sciences de l'information de l'Université de Montréal. Certaines expositions ont été produites lors de stages de durées variées, tandis qu'une autre exposition est le fruit du travail d'un membre du personnel de la DGDA. En fait, la Division a profité de toutes les situations favorables pour mettre en valeur les documents qu'elle conserve.

Plusieurs expositions diffusées par la DGDA ont fait l'objet de partenariats avec des unités de l'Université de Montréal (École de bibliothéconomie et des sciences de l'information, Direction des bibliothèques de I'Université, Herbier Marie-Victorin) ou des organismes externes (Université d'Ottawa, Université McGill, Fondation Museum Nature, Jardin botanique de Montréal). II s'agit ici de partenaires qui ont contribué directement à des expositions soit financièrement, soit par l'ajout de ressources humaines ou de ressources technologiques. Ces collaborations ont permis de réaliser ainsi des expositions de plus grande envergure ou d'offrir une plateforme de diffusion permettant d'accroître le rayonnement des projets. 
Un autre type de contribution a également permis d'enrichir nos expositions virtuelles. II s'agit de l'inclusion de documents provenant d'autres services d'archives. En effet, si plusieurs de nos expositions contiennent exclusivement des documents conservés par la DGDA, d'autres comportent un certain nombre de documents provenant d'autres sources. L'ajout de ces documents nous a permis de bonifier nos expositions, car dans plusieurs cas ils complètent les documents que nous avions. Toutes ces collaborations reposent sur le constat que dans le contexte dans lequel se situent la plupart des services d'archives, nous ne pouvons fonctionner en autarcie et que c'est en travaillant avec les autres que nous sommes plus forts.

\subsection{Les réseaux sociaux}

C'est en 2012 que l'idée d'être présent sur les réseaux sociaux a germé au sein de la DGDA. Étant néophytes en la matière, il nous est apparu judicieux de réaliser au point de départ une analyse d'opportunité. Cette analyse a été réalisée dans le cadre d'un stage de maîtrise de l'École de bibliothéconomie et des sciences de l'information au printemps 2012. Cette analyse nous a donné la possibilité de connaître la forme que prenait la présence de plusieurs services d'archives canadiens sur les réseaux sociaux. Cette analyse nous a permis de déterminer les objectifs et les priorités de la DGDA en ce qui concerne les réseaux sociaux. À l'été 2012, un projet Jeunesse Canada au travail nous a permis de développer des outils et des procédures nous permettant d'amorcer notre présence sur les réseaux sociaux. À l'automne 2012, l'archiviste responsable de la référence a commencé à diffuser de l'information sur les réseaux sociaux. Elle poursuit ce travail avec l'aide ponctuelle de stagiaires. C'est ainsi que depuis 2012, 75 messages ont été diffusés sur Facebook suivi par plus de 500 «fans». Sur Twitter, 334 microbillets ont été envoyés et ont été suivis par plus de 447 abonnés. La DGDA a également diffusé sur Flickr 48 albums et près de 800 photographies. La Division a également une chaîne YouTube diffusant entre autres des vidéos tirées de nos fonds d'archives. La présence de la DGDA sur les réseaux sociaux témoigne de sa volonté d'assurer la diffusion la plus large possible des archives dont elle a la responsabilité.

\subsection{D’autres projets}

La diffusion des archives prend aussi la forme de projets particuliers. En 2012, I'Université de Montréal a mis en place UdeMtélé, un réseau 
d'écrans permettant de joindre les étudiants partout sur le campus. Dès le départ, la DGDA a tenu à être présente sur ce réseau. C'est ainsi que depuis les débuts d'UdeMtélé, la DGDA a diffusé plus d'une trentaine de messages. Les bureaux de la Division de la gestion de documents et des archives sont situés dans le Pavillon Roger-Gaudry dans un corridor assez achalandé. Pour dynamiser le corridor, la DGDA y a fait installer un moniteur diffusant des présentations PowerPoint. Ces présentations abordent différents aspects de l'Histoire de l'Université de Montréal et permettent de présenter un certain nombre de documents d'archives.

Une autre initiative de la responsable de la référence s'inscrit dans cette volonté de faire connaître les documents que nous conservons. Elle envoie régulièrement des communiqués présentant aux doyens et aux bibliothécaires de référence les fonds d'archives pour chacune des disciplines en y spécifiant les fonds actuels, les ajouts et les nouveautés. La DGDA poursuit également sa collaboration avec les étudiants de différents cours de l'École de bibliothéconomie et des sciences de l'information pour des projets de rédaction d'articles, de réalisation d'expositions virtuelles et physiques et d'analyse diplomatique de documents. La Division collabore également à un séminaire de maîtrise en musicologie. Enfin, la DGDA a participé à différents projets de création: résidence d'artiste, projets d'affiche, projet Archives et création (vidéos à partir de pièces d'archives). Ces différentes initiatives illustrent la volonté de la DGDA de trouver de nouvelles avenues pour faire connaître et rendre accessibles les archives dont elle a la garde.

\section{VERS UNE PLUS GRANDE ACCESSIBILITÉ DES DOCUMENTS D'ARCHIVES}

Comme je l'avais indiqué au début de ce texte, les archives définitives, qu'elles soient institutionnelles ou de sources privées, font partie intégrante de la mission de la Division de la gestion de documents et des archives de l'Université de Montréal. Beaucoup de travail a été fait, mais nous ne pouvons pas nous reposer sur nos lauriers. Nous avons des défis auxquels nous avons l'intention de nous attaquer. Nous voulons mieux déterminer les documents institutionnels et privés à conservation permanente. Est-ce que tous les documents versés ou acquis sont véritablement pertinents? Une réflexion s'impose pour en arriver à des règles de conservation encore plus précises et appropriées. Dans un contexte de croissance de l'information, c'est essentiel. En ce qui concerne 
les fonds privés, nous allons mettre à jour notre politique d'acquisition pour qu'elle nous permette de mieux sélectionner les fonds de sources privées qui témoigneront de l'histoire de l'Université de Montréal et qui répondront mieux aux besoins de la recherche.

Nous voulons amorcer une réflexion sur nos méthodes de traitement des documents à conservation permanente. Notre objectif est d'accélérer le traitement des documents pour qu'ils soient accessibles plus rapidement. Nous voulons que les descriptions des archives soient encore plus pertinentes pour en faciliter le repérage. En fait, ce que nous visons, c'est de traiter plus de documents, plus vite et mieux. Nous voulons en arriver à une plus grande accessibilité des archives définitives. Nous allons donc réfléchir aux moyens et aux ressources disponibles pouvant nous permettre de diffuser plus de documents. Nous avons l'intention de réviser notre site Web. Nous allons aussi explorer les possibilités d'utilisation de plateformes de diffusion d'archives. Notre objectif est de permettre aux utilisateurs d'avoir accès à plus de documents que maintenant, que ce soit à partir de notre site Web ou à partir d'une autre source.

Pour relever ces défis, la Division de la gestion de documents et des archives devra être à l'affût des nouvelles tendances en matière de traitement et de diffusion des archives. Mais si le passé est garant de l'avenir, on peut croire que la DGDA trouvera des solutions pour mieux conserver et mieux diffuser le patrimoine archivistique de I'Université de Montréal et pourra ainsi joindre un nombre toujours plus grand d'utilisateurs.

\section{NOTES}

1. UNIVERSITÉ DE MONTRÉAL. DIVISION DE LA GESTION DE DOCUMENTS ET DES ARCHIVES. (2016). Mandat. Repéré à http://www.archiv.umontreal.ca/information/ mandat.html.

2. On peut consulter le texte de la Politique sur la gestion de documents et des archives de I'Université de Montréal à l'adresse suivante: http://secretariatgeneral.umontreal. ca/fileadmin/secretariat/Documents/Reglements/adm10 49-Politique gestion documents archives.pdf

3. Depuis 2008, la numérisation a remplacé le microfilmage des documents qui auparavant faisait partie du traitement définitif.

4. Les notices descriptives et les termes d'indexation sont saisis dans le logiciel SyGED. 